\title{
On the effect of functionally graded materials on resonances of rotating beams
}

\author{
Arnaldo J. Mazzei, Jr.* \\ C.S. Mott Engineering and Science Center, Department of Mechanical Engineering, Kettering University, Flint, \\ MI, USA
}

Received 11 February 2010

Revised 15 November 2011

\begin{abstract}
Radially rotating beams attached to a rigid stem occur in several important engineering applications. Some examples include helicopter blades, turbine blades and certain aerospace applications. In most studies the beams have been treated as homogeneous. Here, with a goal of system improvement, non-homogeneous beams made of functionally graded materials are explored. The effects on the natural frequencies of the system are investigated. Euler-Bernoulli theory, including an axial stiffening effect and variations of both Young's modulus and density, is employed. An assumed mode approach is utilized, with the modes taken to be beam characteristic orthogonal polynomials. Results are obtained via Rayleigh-Ritz method and are compared for both the homogeneous and non-homogeneous cases. It was found, for example, that allowing Young's modulus and density to vary by approximately 2.15 and 1.15 times, respectively, leads to an increase of $23 \%$ in the lowest bending rotating natural frequency of the beam.
\end{abstract}

Keywords: Axially rotating beams, FGM, spatially varying material properties

\section{Nomenclature}

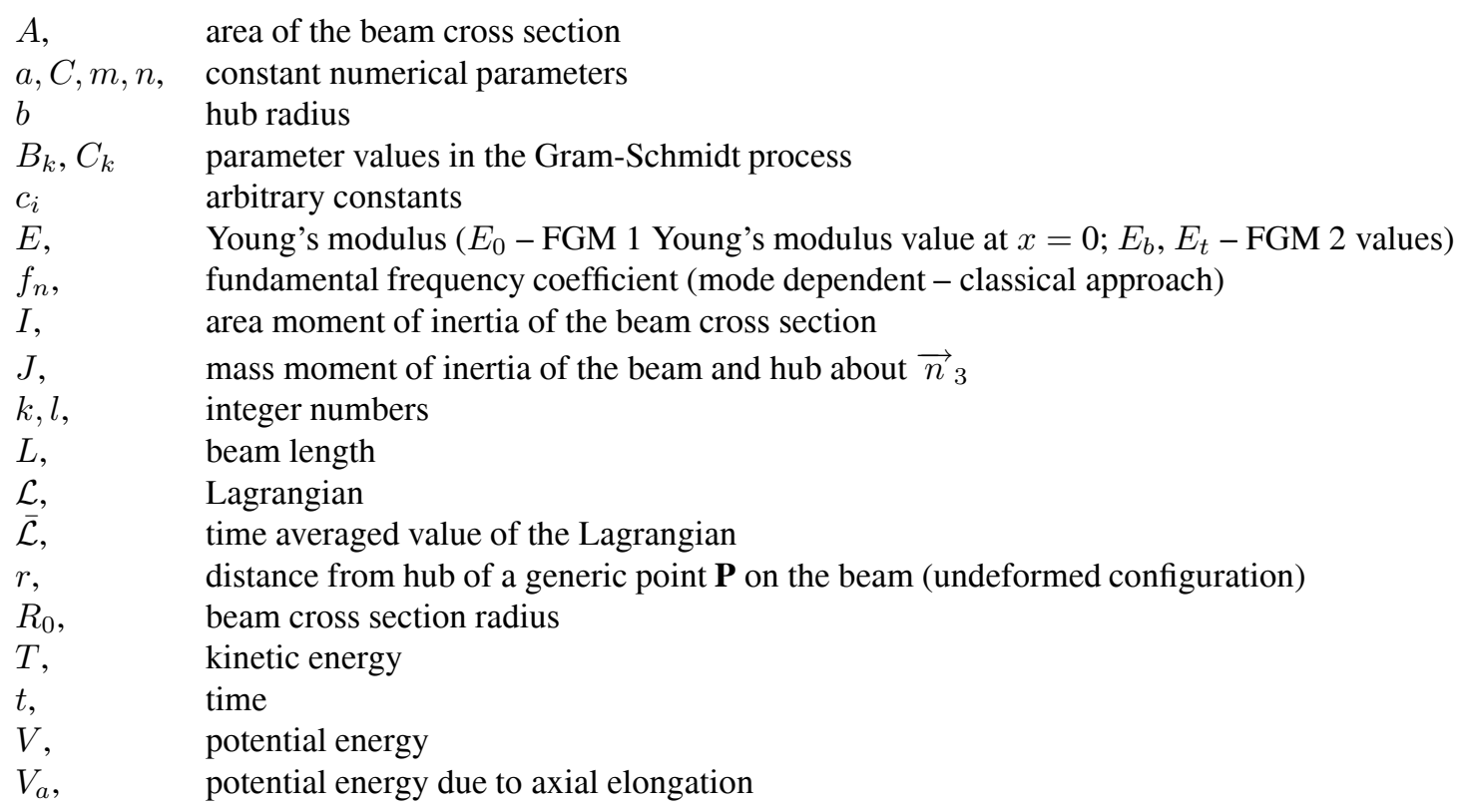

${ }^{*}$ Corresponding author. Tel.: +1810762 7878; Fax: +1 810762 7860; E-mail: amazzei@ kettering.edu. 


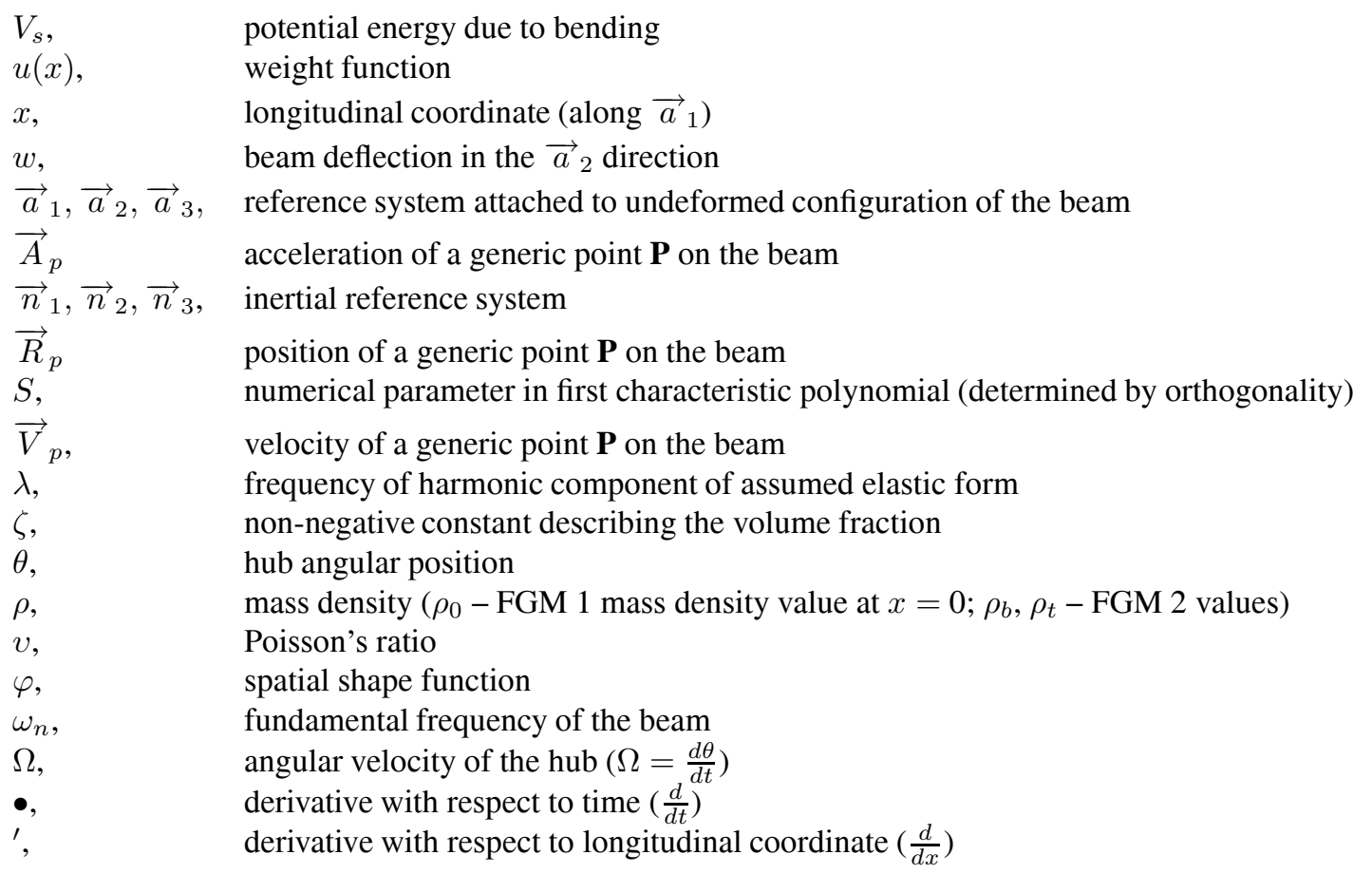

\section{Introduction}

Rotating machinery form an important part of engineering and radially rotating beams constitute a major category of such systems. Some examples include rotor blades, propellers and turbines. For vibration control it is important to identify possible system resonances and, if required and possible, change these values.

Extensive work on these types of problems has been done in the aerospace literature. Comprehensive reviews can be found in the papers of Kane and Ryan [13] and Haering et al. [7]. They, and others, showed that at high speeds the rotating structure can be prone to instabilities. It is assumed here that the rotational speeds are small enough that no instabilities are encountered.

There are numerous works on vibrations of radially rotating beams (uniform beams, beams including pre-twist and tapered beams). Two classes of problems arise, namely, prescribed motions and prescribed torques. Earlier studies on the former type of problem can be found in the texts by Putter and Manor [24], Hoa [8], Hodges [9] and Hodges and Rutkwoski [10]. Putter and Manor used a finite element approach to obtain the natural frequencies and mode shapes of the beam, including shearing forces, rotary inertia and varying centrifugal forces. Hoa also utilized a finite element approach for the same objective, but effects of root radius, setting angle and tip mass were included. Hodges used asymptotic expansions to obtain an approximate value for the fundamental frequency of a uniform beam and, in a subsequent work, Hodges and Rutkwoski used a finite element approach to calculate the eigenvalues and eigenvectors of the beam including different hub radii, tapered beams and beams with discontinuities. Kojima [15] investigated the transient flexural vibrations of a beam/mass system attached to a rotating rigid body.

The prescribed torque problem has been studied by, for example, Yigit et al. [28]. In that work the flexural motion of a rotating beam was investigated by using a specified torque profile to drive the rotating body (so that the rigid body motion was not known a priori). The results showed that for small values of the ratio of the flexible beam and rigid shaft inertia, uncoupled equations could lead to substantially incorrect results, notably with regard to frequencies.

Lee et al. [16] presented experimental results confirming that centrifugal effects cannot be neglected, even at first order, when modeling these systems.

Models utilizing a Timoshenko beam type approach (other than Euler-Bernoulli) are also numerous. See, for example, the work of Lin and Hsiao [19] which investigates the effect of Coriolis force on the natural frequencies 
of the rotating beam. More complex models including base excitation can be found in references $[12,26]$. Also, the fundamental frequency of rotating beams with pre-twist was studied by, for example, Hu et al. [11].

Beams made of Functionally Graded Materials (FGM - see, for example, reference [21]) and rotating about their longitudinal axes have recently received attention. Oh et al. [22] investigated the thermoelastic modeling and behavior of circular cylindrical thin-walled beams made of FGMs and spinning with constant speed about their longitudinal axes. The implications of conservative and gyroscopic forces in conjunction with a temperature field, which yielded material degradation of the beam elastic properties on their vibration and instability, were studied. The FGMs considered utilized a continuously graded variation in the composition of the ceramic and metal phases, across the beam wall thickness, by means of a simple power law distribution. Results showed that the spinning beam could experience instabilities by flutter and divergence and that the volume fraction played a strong role. Ahmad and Naeem [1] investigated the vibration characteristics of longitudinally rotating FGM cylindrical shells for a number of distinct boundary conditions. The equations of motion were obtained using thin shell theory and were solved using a wave propagation approach. Numerical results were compared to ones available in the literature and were found to be in a good agreement.

The role of FGMs in radially rotating beams has not been fully investigated. Fazelzadeh and Hosseini [5] studied a thin-walled FGM beam that could be used as a rotating blade in turbo-machinery under aero-thermoelastic loading. The governing equations were based on first order shear deformation theory and included the effects of presetting angle, secondary warping, temperature gradient through the wall thickness of the beam and rotational speed. Results demonstrated that the natural frequencies of the system decreased under aero-thermoelastic loading at high mach numbers.

Librescu et al. [18], in a prescribed motion problem, studied the effects of material variation through the beam thickness on the eigenfrequencies of the system. In that context, the implications on the eigenfrequencies and mode shapes of the proportions of the two phases of the FGM (ceramic and metal) were discussed and noted. The structural model developed was found to be useful in the design of advanced rotor blades made up of FGMs and exposed to high-temperature gradients.

In a recent study, Piovan and Sampaio [23] developed a non-linear model of a rotating beam including shear deformation and metallic-ceramic FGMs. The FGM properties varied along the thickness of the beam in a metallicrich core/ceramic-rich surfaces distribution. Assuming prescribed motion, a finite element approach was employed to investigate some features of the problem. It was found that the ratio between constituents plays an important role on the dynamic behavior of the beam. This was observed to be important when the beam was mainly composed of metal and subjected to sudden changes in acceleration.

In the present work a prescribed motion problem is investigated with material properties varying along the length of the beam. The possibility of changing the natural frequencies of vibration of a cantilever radially rotating beam is investigated. The approach includes changing the material of the beam from a homogeneous type to a FGM while keeping the physical dimensions of the beam constant. An assumed mode approach is employed with the mode shapes taken to be beam characteristic orthogonal polynomials. Results for the frequencies are obtained via Rayleigh-Ritz method with the aid of MAPLE ${ }^{\circledR}[30]$.

Results indicate that, for example, in the case of a beam made of aluminum and silicon carbide, the frequencies can be increased substantially. For instance, when the rotational speed is approximately $955 \mathrm{rpm}$, the increase for the first mode frequency is approximately $23 \%$ when compared to a pure aluminum beam. Higher modes show percentual increases even higher, on the order of $40 \%$.

\section{Modeling}

Figure 1 shows a beam with length $L$ attached to a rigid hub of radius $b$. The hub rotates radially with angular velocity $\Omega$. A set of mutually perpendicular unit vectors $\vec{a}_{1}, \vec{a}_{2}$ and $\vec{a}_{3}$ is attached to the undeformed configuration of the beam. A second set of mutually perpendicular unit vectors, $\vec{n}_{1}, \vec{n}_{2}$ and $\vec{n}_{3}$ is assumed to be the inertial reference frame. The vector $\vec{n}_{3}$ is the axis of rotation for the hub and remains parallel to $\vec{a}_{3}$ during motion. $\theta$ is the angle between the vector $\vec{n}_{1}$ and $\vec{a}_{1}$ and defines the angular position of the hub with respect to the inertial frame. Note that $\Omega=\frac{d \theta}{d t}$. 


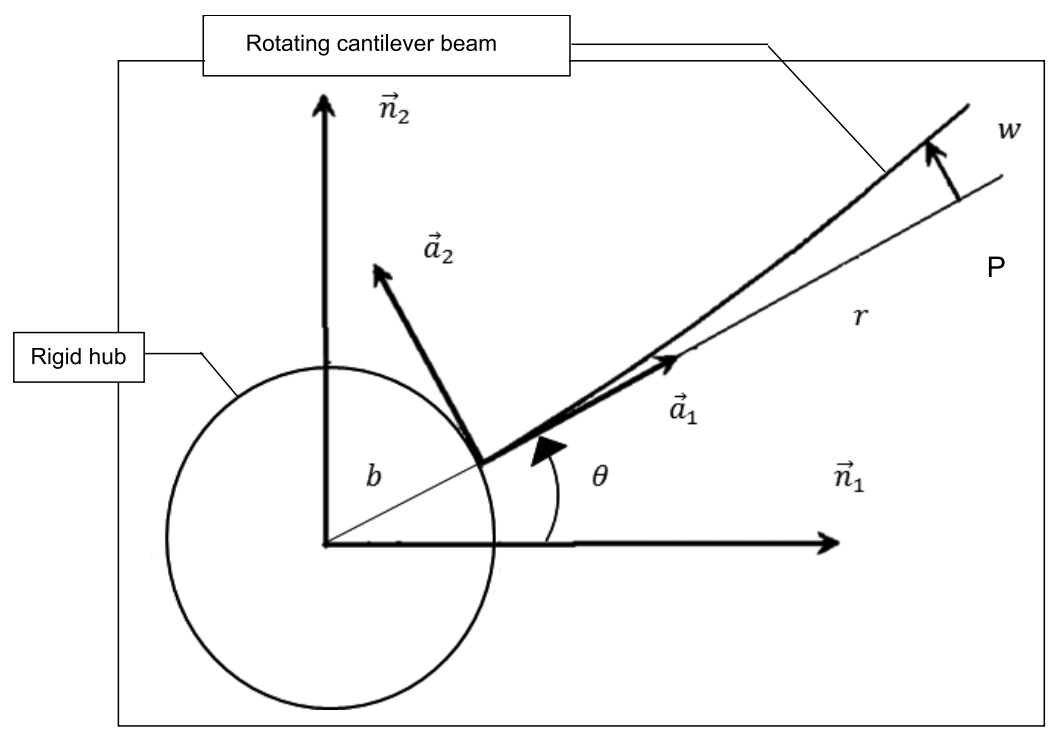

Fig. 1. Rotating cantilever beam.

For rotation on the plane, the position, velocity and acceleration of point $\mathbf{P}$ on the deformed configuration of the beam are given, respectively, by:

$$
\begin{aligned}
& \overrightarrow{R_{p}}=(b+r) \vec{a}_{1}+w \vec{a}_{2} \\
& \overrightarrow{V_{p}}=-w \dot{\theta} \vec{a}_{1}+[(b+r) \dot{\theta}+\dot{w}] \vec{a}_{2} \\
& \overrightarrow{A_{p}}=-\left[2 \dot{w} \dot{\theta}+(b+r) \dot{\theta^{2}}+w \ddot{\theta}\right] \vec{a}_{1}+\left[\ddot{w}+(b+r) \ddot{\theta}-w \dot{\theta}^{2}\right] \vec{a}_{2}
\end{aligned}
$$

The kinetic energy of the beam can be computed from (note $r=x$ ):

$$
T=\frac{1}{2} \int_{0}^{L} \rho(x) A\left\{\left(\frac{\partial w}{\partial t}\right)^{2}+2 \dot{\theta}\left(\frac{\partial w}{\partial t}\right)(b+x)+\dot{\theta}^{2}\left[(b+x)^{2}+w^{2}\right]\right\} d x+\frac{1}{2} J \dot{\theta}^{2}
$$

The potential energy of the system comes from two parts. The first part is caused by the bending elastic strain. Using Euler-Bernoulli beam theory, this can be calculated by:

$$
V_{s}=\frac{1}{2} \int_{0}^{L} E(x) I\left\{\left(\frac{\partial^{2} w}{\partial x^{2}}\right)^{2}\right\} d x
$$

The second part is due to the centrifugal force acting on the beam, which causes axial elongation (see, for example, reference [26]). It is given by:

$$
V_{a}=\frac{1}{2} \int_{0}^{L}\left(\frac{\partial w}{\partial x}\right)^{2}\left\{\int_{x}^{L} \rho(x) A\left[\dot{\theta}^{2}(b+x)+2 \dot{\theta} \frac{\partial w}{\partial t}+w \ddot{\theta}\right] d x\right\} d x
$$

The total potential energy is obtained from:

$$
V=V_{s}+V_{a}
$$

Next an assumed mode approach is adopted. The following is assumed for the elastic form (harmonic motion):

$$
w(x, t)=\varphi(x) \cos (\lambda t)
$$

where: 


$$
\varphi(x)=\sum_{1}^{k} c_{i} \phi_{i}(x)
$$

The shape function $\varphi(x)$ is taken to be a linear combination of $k$ beam characteristic orthogonal polynomials $\phi_{i}$ where the $c_{i}$ are arbitrary constants to be determined. Each polynomial satisfies the geometric boundary conditions at the clamped end of the beam.

The set of orthogonal polynomials is generated by the Gram-Schmidt process [3] as demonstrated by Bhat [2]. The procedure is reproduced below.

The first polynomial is chosen as the simplest one of least order that satisfies both the geometrical and natural boundary conditions of the beam. The other members of the orthogonal set in the interval $x_{1}<x<x_{2}$ are generated by using:

$$
\phi_{2}(x)=\left(x-B_{2}\right) \phi_{1}(x), \ldots, \phi_{k}(x)=\left(x-B_{k}\right) \phi_{k-1}(x)-\left(C_{k}\right) \phi_{k-2}(x)
$$

where:

$$
B_{k}=\frac{\left[\int_{x_{1}}^{x_{2}} x u(x)\left(\phi_{k-1}(x)\right)^{2} d x\right]}{\left[\int_{x_{1}}^{x_{2}} u(x)\left(\phi_{k-1}(x)\right)^{2} d x\right]}
$$

and

$$
C_{k}=\frac{\left[\int_{x_{1}}^{x_{2}} x u(x)\left(\phi_{k-1}(x) \phi_{k-2}(x)\right) d x\right]}{\left[\int_{x_{1}}^{x_{2}} u(x)\left(\phi_{k-2}(x)\right)^{2} d x\right]}
$$

The function $u(x)$ is the weight function and, in the following, is assumed to be 1 . Note that the polynomials $\phi_{k}$ satisfy the orthogonality condition:

$$
\int_{x_{1}}^{x_{2}} u(x)\left(\phi_{k}(x) \phi_{l}(x)\right) d x= \begin{cases}0, & k \neq l \\ a_{k l}, & k=l\end{cases}
$$

For the present problem, as done by Bhat [2], the first polynomial is taken to satisfy only the two geometrical boundary conditions at the clamped end. It is assumed to have the following form:

$$
\phi_{1}(x)=S x^{2}
$$

The constant $S$ is chosen so that Eq. (13) is satisfied in the following manner:

$$
\int_{0}^{L}\left(\phi_{k}(x)\right)^{2} d x=1
$$

Next the Lagrangian [6] can be written as:

$$
\begin{aligned}
& \mathcal{L}=T-V=\frac{1}{2}\left\{\int_{0}^{L} \rho(x) A\left\{\varphi^{2} \dot{\eta}^{2}+2 \dot{\theta} \varphi \dot{\eta}(b+x)+\dot{\theta}^{2}\left[(b+x)^{2}+\varphi^{2} \eta^{2}\right]\right\} d x\right\}+\frac{1}{2} J \dot{\theta}^{2} \\
& -\frac{1}{2} \int_{0}^{L}\left\{E(x) I \varphi^{\prime \prime 2} \eta^{2}+\varphi^{\prime 2} \eta^{2}\left\{\int_{x}^{L} \rho(x) A\left[\dot{\theta}^{2}(b+x)+2 \dot{\theta} \varphi \dot{\eta}+\varphi \eta \ddot{\theta}\right] d x\right\}\right\} d x
\end{aligned}
$$

and the time averaged value of the Lagrangian can be obtained from:

$$
\overline{\mathcal{L}}=\int_{0}^{\frac{2 \pi}{\lambda}} \mathcal{L} d t
$$

Utilizing Eqs (8), (9), (16) and (17) and applying the Rayleigh-Ritz method, one obtains the following homogeneous simultaneous equations:

$$
\frac{\partial \overline{\mathcal{L}}}{\partial c_{i}}=0, \quad i=1,2,3 \ldots k
$$

which can be cast into a standard eigenvalue problem form, from which the natural frequencies can be calculated.

In the following, homogeneous and non-homogeneous material types are considered. The non-homogeneous materials utilized are FGMs. Results for the fundamental frequencies of the FGM beams are compared to those for the homogeneous beams. 
Table 1

Material properties for Al/SiC FGM

\begin{tabular}{lc}
\hline \multicolumn{2}{c}{ Aluminum/Silicon carbide } \\
\hline$E_{0}(\mathrm{GPa})$ & 105.197 \\
$\rho_{0}\left(\mathrm{~kg} / \mathrm{m}^{3}\right)$ & 2710.000 \\
$a$ & 1.14568 \\
$m$ & 1.00000 \\
$n$ & 0.17611 \\
\hline
\end{tabular}

Table 2

Homogeneous material properties

\begin{tabular}{lcccc}
\hline & Steel & Aluminum & Zirconium Oxide & Aluminum Oxide \\
\hline$E_{0}(\mathrm{GPa})$ & 210 & 71 & 151 & 380 \\
$\rho_{0}\left(\mathrm{~kg} / \mathrm{m}^{3}\right)$ & 7850 & 2710 & 6000 & 3690 \\
$v$ & 0.3 & 0.3 & 0.3 & 0.3 \\
\hline
\end{tabular}

Table 3

Convergence of natural frequencies as a function of number of terms in the polynomial shape function - Aluminum beam, angular velocity $=0$

\begin{tabular}{cccccc}
\hline Number of terms & Mode 1 $(\mathrm{Hz})$ & Mode 2 $(\mathrm{Hz})$ & Mod 3 $(\mathrm{Hz})$ & Mode 4 $(\mathrm{Hz})$ & Mode 5 $(\mathrm{Hz})$ \\
\hline 2 & 22.76 & 224.28 & & & \\
3 & 22.66 & 143.26 & 761.26 & & \\
4 & 22.66 & 142.77 & 408.17 & 1814.45 & \\
5 & 22.66 & 141.98 & 407.49 & 828.42 & 3628.86 \\
6 & 22.66 & 141.98 & 397.67 & 827.69 & 1456.67 \\
7 & 22.66 & 141.98 & 397.67 & 776.26 & 1456.14 \\
\hline
\end{tabular}

\section{Numerical results}

\subsection{FGM 1 model}

The FGM model used here is based on the one described by Chiu and Erdogan [29]. The material is assumed to be isotropic and non-homogeneous with properties given by:

$$
E(x)=E_{0}\left(a \frac{x}{L}+1\right)^{m}, \rho(x)=\rho_{0}\left(a \frac{x}{L}+1\right)^{n}
$$

where $a, m$ and $n$ are arbitrary real constants with $a>-1 . E_{0}$ and $\rho_{0}$ are the Young's modulus and mass density at $x=0$. The material is a composite made from aluminum and silicon carbide ( $\mathrm{Al} / \mathrm{SiC})$. Its properties are given in Table 1 and are taken from reference [29].

For the numerical simulations, the beam is taken to have a circular cross section with a radius given by $R_{0}=$ $0.0127 \mathrm{~m}$. The length of the beam is $L=0.896 \mathrm{~m}$ and the radius of the hub is $b=0.05 \mathrm{~m}$. Also, the mass moments of inertia of the rigid and flexible parts are taken to be related in the following manner: $J=(0.5)\left(\int_{0}^{L} \rho(x) A(b+x)^{2} d x\right)$ ("inertia ratio" $=0.5$ ). The homogeneous materials properties are given in Table 2.

Using Eq. (18), the natural frequencies are evaluated for the beams at two different rotational speeds, namely, $\Omega=$ 0 and $\Omega=100 \mathrm{rad} / \mathrm{s}$ (approximately $955 \mathrm{rpm}$ ). Results are presented in Tables 3 to 6 . Convergence is checked by varying the number of terms included in the polynomial shape function.

For comparison purposes, the natural frequencies of the non-rotating aluminum beam can be calculated using traditional approaches by (see, for example [20]):

$$
\omega_{n}=f_{n} \sqrt{\frac{E I}{\rho A L^{4}}}
$$

where $f_{n}$ is a numerical value specific to each mode of vibration. For instance, for the first mode, $f_{n}=f_{1}=3.5160$, for the second mode $f_{2}=22.0345$ and for the third mode $f_{3}=61.6972$. This gives: $\omega_{1}=22.66 \mathrm{~Hz}, \omega_{2}=141.98$ $\mathrm{Hz}$ and $\omega_{3}=397.55 \mathrm{~Hz}$. 
Table 4

Convergence of natural frequencies as a function of number of terms in the polynomial shape function - Aluminum beam, angular velocity $=100 \mathrm{rad} / \mathrm{s}$

\begin{tabular}{cccccc}
\hline Number of terms & Mode 1 $(\mathrm{Hz})$ & Mode 2 $(\mathrm{Hz})$ & Mod 3 $(\mathrm{Hz})$ & Mode 4 $(\mathrm{Hz})$ & Mode 5 $(\mathrm{Hz})$ \\
\hline 2 & 24.36 & 227.66 & & & \\
3 & 24.14 & 148.36 & 763.87 & & \\
4 & 24.14 & 147.88 & 413.51 & 1816.44 & \\
5 & 24.14 & 147.21 & 412.90 & 833.78 & 3630.99 \\
6 & 24.14 & 147.21 & 403.44 & 833.15 & 1461.91 \\
7 & 24.14 & 147.21 & 403.42 & 782.33 & 1461.38 \\
\hline
\end{tabular}

Table 5

Convergence of natural frequencies as a function of number of terms in the polynomial shape function $-\mathrm{Al} / \mathrm{SiC}$ beam, angular velocity $=0$

\begin{tabular}{cccccc}
\hline Number of terms & Mode 1 $(\mathrm{Hz})$ & Mode 2(Hz) & Mod 3(Hz) & Mode 4(Hz) & Mode 5(Hz) \\
\hline 2 & 29.23 & 357.26 & & & \\
3 & 28.52 & 203.73 & 1246.84 & & \\
4 & 28.52 & 197.94 & 595.36 & 3004.20 & \\
5 & 28.52 & 197.65 & 576.84 & 1217.24 & 6035.23 \\
6 & 28.52 & 197.65 & 569.12 & 1169.54 & 2129.85 \\
7 & 28.52 & 197.65 & 568.71 & 1126.17 & 2020.73 \\
\hline
\end{tabular}

Table 6

Convergence of natural frequencies as a function of number of terms in the polynomial shape function $-\mathrm{Al} / \mathrm{SiC}$ beam, angular velocity $=100 \mathrm{rad} / \mathrm{s}$

\begin{tabular}{cccccc}
\hline Number of terms & Mode 1 $(\mathrm{Hz})$ & Mode 2(Hz) & Mod 3(Hz) & Mode 4(Hz) & Mode 5(Hz) \\
\hline 2 & 30.47 & 359.44 & & & \\
3 & 29.61 & 207.46 & 1248.47 & & \\
4 & 29.59 & 201.82 & 599.16 & 3005.43 & \\
5 & 29.59 & 210.56 & 581.05 & 1220.93 & 6036.16 \\
6 & 29.59 & 210.56 & 573.41 & 1173.93 & 2133.12 \\
7 & 29.59 & 210.56 & 573.41 & 1130.75 & 2024.92 \\
\hline
\end{tabular}

Some observations can then be made. The tables demonstrate that convergence for the first three modes can be achieved with the use of seven terms in the polynomial shape function. As seen in Table 3, excellent agreement between the frequencies calculated above and the ones given by the approach was obtained, lending confidence to the method.

A comparison between values given in Tables 3 and 4 shows the stiffening effect that the rotational speed introduces into the system. The same can be said for the FGM shaft (Table 5 versus Table 6).

The use of the FGM led to increases in all the frequencies. For the non-rotating case the first frequency increases by $26 \%$, the second by $39 \%$ and the third by $43 \%$. For the rotating case, the first frequency increases by $23 \%$, the second by $43 \%$ and the third by $42 \%$. These results demonstrate that the use of this FGM can be beneficial for the application when the goal is to increase resonant frequencies.

Moreover, note that the frequency increases were obtained via a material with variations of Young's modulus and density, along the length of the beam, of 2.15 and 1.15 times, respectively. When compared to the aluminum beam, the increase in weight is only about $8 \%$. Here these values are considered reasonable and the observed trends are beneficial.

\subsection{FGM 2 model}

The second FGM model is developed by assuming that its composition is derived from of a mixture of two materials, with the material variation given by a power-law gradient (see, for instance, reference [17]). The effective properties are given by:

$$
E(x)=E_{b}+\left(E_{t}-E_{b}\right)\left(\frac{x}{L}\right)^{\zeta}, \quad \rho(x)=\rho_{b}+\left(\rho_{t}-\rho_{b}\right)\left(\frac{x}{L}\right)^{\zeta}
$$


Table 7

Convergence of natural frequencies as a function of number of terms in the polynomial shape function $\mathrm{Al}_{2} \mathrm{O}_{3} / \mathrm{Al}$ beam, angular velocity $=0$

\begin{tabular}{cccccc}
\hline Number of terms & Mode 1 $(\mathrm{Hz})$ & Mode 2 $(\mathrm{Hz})$ & Mod 3 $(\mathrm{Hz})$ & Mode 4 $(\mathrm{Hz})$ & Mode 5 $(\mathrm{Hz})$ \\
\hline 2 & 45.93181463 & 296.8184430 & & & \\
3 & 45.89431680 & 246.7079979 & 895.6802700 & & \\
4 & 45.88942898 & 243.9079402 & 672.1557060 & 2005.775835 & \\
5 & 45.88805364 & 243.5383077 & 648.3818570 & 1359.628762 & 3861.039990 \\
6 & 45.88789314 & 243.4116960 & 644.1356000 & 1267.198320 & 2417.326605 \\
7 & 45.88786638 & 243.4053993 & 642.2768530 & 1251.160955 & 2133.862662 \\
\hline
\end{tabular}

Table 8

Convergence of natural frequencies as a function of number of terms in the polynomial shape function $\mathrm{Al}_{2} \mathrm{O}_{3} / \mathrm{Al}$ beam, angular velocity $=100 \mathrm{rad} / \mathrm{s}$

\begin{tabular}{cccccc}
\hline Number of terms & Mode 1 $(\mathrm{Hz})$ & Mode 2 $(\mathrm{Hz})$ & Mod 3 $(\mathrm{Hz})$ & Mode 4 $(\mathrm{Hz})$ & Mode 5 $(\mathrm{Hz})$ \\
\hline 2 & 46.80055954 & 299.2361516 & & & \\
3 & 46.78446580 & 249.4685694 & 897.7694340 & & \\
4 & 46.78139328 & 246.7568208 & 675.0733720 & 2007.481776 & \\
5 & 46.78025050 & 246.4140287 & 651.4061420 & 1362.469208 & 3861.764011 \\
6 & 46.78009469 & 246.2899516 & 647.2391395 & 1269.562755 & 2380.346332 \\
7 & 46.78006850 & 246.2833426 & 645.3639115 & 1239.962126 & 1959.611411 \\
\hline
\end{tabular}

Table 9

Convergence of natural frequencies as a function of number of terms in the polynomial shape function $\mathrm{ZrO}_{2} / \mathrm{Al}$ beam, angular velocity $=0$

\begin{tabular}{cccccc}
\hline Number of terms & Mode 1 $(\mathrm{Hz})$ & Mode 2 $(\mathrm{Hz})$ & Mod 3 $(\mathrm{Hz})$ & Mode 4 $(\mathrm{Hz})$ & Mode 5 $(\mathrm{Hz})$ \\
\hline 2 & 28.08988951 & 214.5945200 & & & \\
3 & 28.08297948 & 151.4218537 & 698.1197095 & & \\
4 & 28.06952986 & 151.4111546 & 412.8852145 & 1650.720092 & \\
5 & 28.06911403 & 150.5151688 & 412.4049606 & 827.9034520 & 3304.298998 \\
6 & 28.06911260 & 150.4936100 & 402.6999892 & 825.4927805 & 1471.638531 \\
7 & 28.06911238 & 150.4924530 & 402.5012276 & 784.7620085 & 1424.763892 \\
\hline
\end{tabular}

Table 10

Convergence of natural frequencies as a function of number of terms in the polynomial shape function $\mathrm{ZrO}_{2} / \mathrm{Al}$ beam, angular velocity $=100 \mathrm{rad} / \mathrm{s}$

\begin{tabular}{cccccc}
\hline Number of terms & Mode 1 $(\mathrm{Hz})$ & Mode 2 $(\mathrm{Hz})$ & Mod 3 $(\mathrm{Hz})$ & Mode 4 $(\mathrm{Hz})$ & Mode 5 $(\mathrm{Hz})$ \\
\hline 2 & 29.45030348 & 217.6377362 & & & \\
3 & 29.40626868 & 155.4756157 & 700.5396945 & & \\
4 & 29.40265500 & 155.4688272 & 417.1733580 & 1652.561670 & \\
5 & 29.40216803 & 154.6751698 & 416.6782194 & 831.9742340 & 3303.099721 \\
6 & 29.40216054 & 154.6504006 & 407.2461077 & 829.7066525 & 1432.472568 \\
7 & 29.40216034 & 154.6496372 & 407.0718360 & 778.1836840 & 1430.743812 \\
\hline
\end{tabular}

where $\zeta$ is a positive constant describing the volume fraction, which can be determined experimentally [17]. The subscripts $\mathbf{b}$ and $\mathbf{t}$ refer to the value of the parameter at $x=0$ and $x=\mathrm{L}$, respectively. These values are the ones for the "pure" materials involved in the composition of the FGM.

In the following the FGMs are taken to be composed of two phases, metallic and ceramic, as in the example above, but with the distinct variations given by Eq. (21). A brief discussion on the manufacturing processes for such materials can be found in reference [25] (see also reference [27] for a different type of application of these materials). For the examples discussed below, $\zeta=1$ is used and the pure material properties are given in Table 2. (A study on the effects of $\zeta$ on the frequencies and weight of the shafts is carried out later.)

For the first example the beam is assumed to be aluminum oxide (alumina $-\mathrm{Al}_{2} \mathrm{O}_{3}$ ) "rich" at $x=0$ and aluminum "rich" at $x=L$. Tables 7 and 8 demonstrate that convergence for the first three modes can be achieved with the use of seven terms. A comparison between the values given in the tables highlights the stiffening effect due to the rotational speed, as observed above for both the aluminum and $\mathrm{Al} / \mathrm{SiC}$ shafts. 

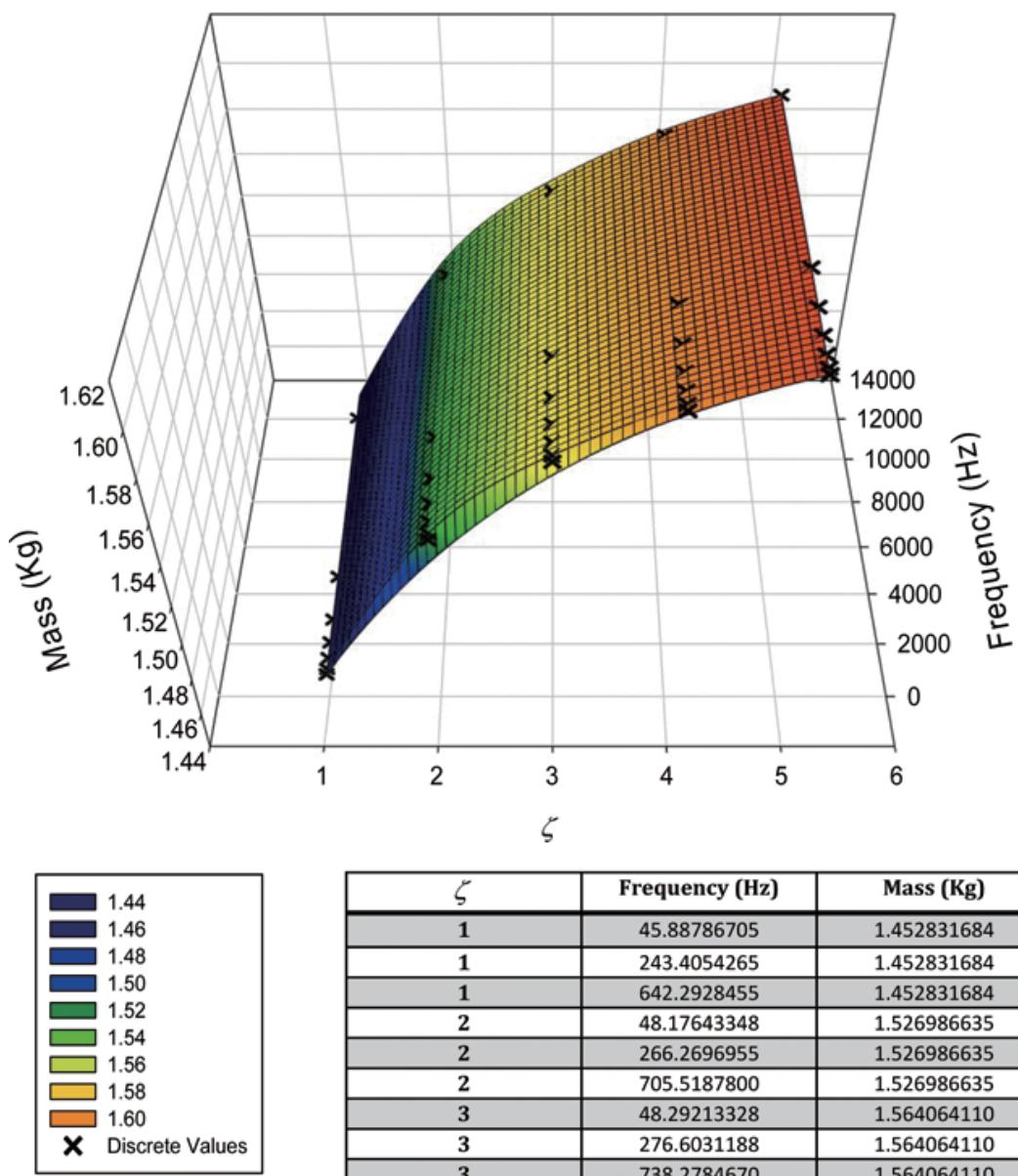

\begin{tabular}{|c|c|c|}
\hline S & Frequency (Hz) & Mass (Kg) \\
\hline $\mathbf{1}$ & 45.88786705 & 1.452831684 \\
\hline $\mathbf{1}$ & 243.4054265 & 1.452831684 \\
\hline $\mathbf{1}$ & 642.2928455 & 1.452831684 \\
\hline $\mathbf{2}$ & 48.17643348 & 1.526986635 \\
\hline $\mathbf{2}$ & 266.2696955 & 1.526986635 \\
\hline $\mathbf{3}$ & 705.5187800 & 1.526986635 \\
\hline $\mathbf{3}$ & 48.29213328 & 1.564064110 \\
\hline $\mathbf{3}$ & 276.6031188 & 1.564064110 \\
\hline $\mathbf{4}$ & 738.2784670 & 1.564064110 \\
\hline $\mathbf{4}$ & 48.04091276 & 1.586310595 \\
\hline $\mathbf{4}$ & 281.9085360 & 1.586310595 \\
\hline $\mathbf{5}$ & 758.0105165 & 1.586310595 \\
\hline $\mathbf{5}$ & 47.74754685 & 1.601141585 \\
\hline $\mathbf{5}$ & 284.7529364 & 1.601141585 \\
\hline & 770.7616535 & 1.601141585 \\
\hline
\end{tabular}

Fig. 2. Mass and frequency variation as a function of $\zeta$ for $\mathrm{Al}_{2} \mathrm{O}_{3}-\mathrm{Al}$.

Increases in frequencies, when compared to the aluminum shaft, are as follows. For the non-rotating case the first frequency increases by $103 \%$, the second by $71 \%$ and the third by $62 \%$. For the rotating case, the first frequency increases by $94 \%$, the second by $67 \%$ and the third by $60 \%$. Note that this FGM can lead to significant increases in the resonant frequencies. The FGM increases the overall weight of the shaft by about $18 \%$ when compared to the homogeneous material.

For comparison purposes within the same material category, a second beam composed by zirconium dioxide (zirconia $\left.-\mathrm{ZrO}_{2}\right)$ and aluminum is considered next $\left(\mathrm{ZrO}_{2}\right.$ "rich" at $x=0$ and aluminum "rich" at $\left.x=L\right)$. For this case, convergence was also observed to occur with the use of seven terms, as well as, the rotational stiffening effect noticed in the previous examples. Tables 9 and 10 show the frequencies for the two distinct velocities.

For this case increases in frequencies when compared to the aluminum shaft are: $24 \%$ (first frequency), $6 \%$ (second frequency) and 1\% (third frequency) for the non-rotating case. For the rotating case these are: $22 \%, 5 \%$ and $1 \%$, respectively. For this FGM the overall weight increase is about $61 \%$ when compared to the homogeneous shaft.

It is seen that the choice of the ceramic phase in this FGM model has a strong effect both on the resonant frequencies and overall weight of the shaft. 

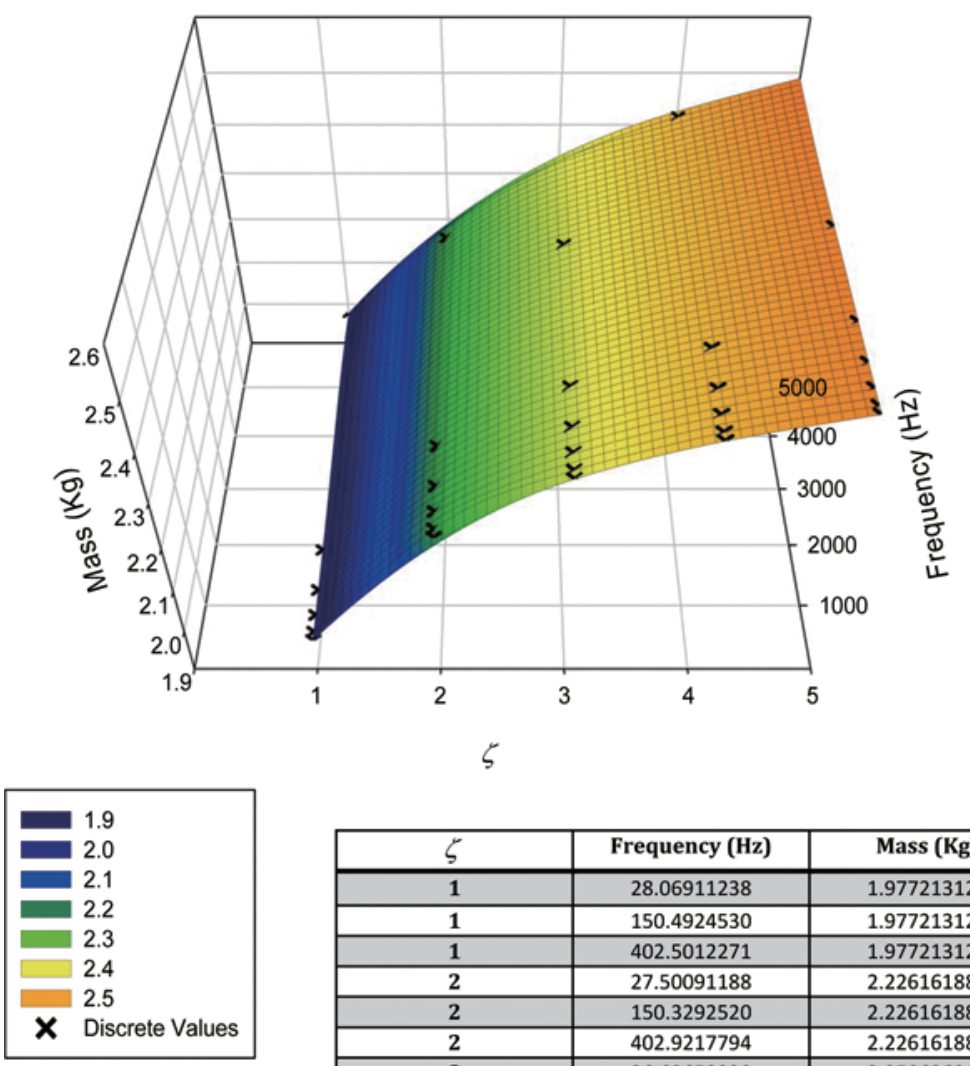

\begin{tabular}{|c|c|c|}
\hline $\boldsymbol{\zeta}$ & Frequency (Hz) & Mass (Kg) \\
\hline $\mathbf{1}$ & 28.06911238 & 1.977213120 \\
\hline $\mathbf{1}$ & 150.4924530 & 1.977213120 \\
\hline $\mathbf{1}$ & 402.5012271 & 1.977213120 \\
\hline $\mathbf{2}$ & 27.50091188 & 2.226161883 \\
\hline $\mathbf{2}$ & 150.3292520 & 2.226161883 \\
\hline $\mathbf{2}$ & 402.9217794 & 2.226161883 \\
\hline $\mathbf{3}$ & 26.69658930 & 2.350636264 \\
\hline $\mathbf{3}$ & 150.1542518 & 2.350636264 \\
\hline $\mathbf{3}$ & 404.3161344 & 2.350636264 \\
\hline $\mathbf{4}$ & 26.04567336 & 2.425320893 \\
\hline $\mathbf{4}$ & 149.8692571 & 2.425320893 \\
\hline $\mathbf{4}$ & 405.4614045 & 2.425320893 \\
\hline $\mathbf{5}$ & 25.54265454 & 2.475110645 \\
\hline $\mathbf{5}$ & 149.4884617 & 2.475110645 \\
\hline $\mathbf{5}$ & 406.1527234 & 2.475110645 \\
\hline
\end{tabular}

Fig. 3. Mass and frequency variation as a function of $\zeta$ for $\mathrm{ZrO}_{2}-\mathrm{Al}$.

It is also possible to utilize the same FGM model Eq. (21) for the case of two distinct metallic phases in the composition of the FGM shaft (see reference [17]). As a final comparison study, consider a shaft made of steel and aluminum (steel "rich" at $x=0$ and aluminum "rich" at $x=L$ ). Properties for structural steel are given in Table 2.

In this case the FGM leads to the following results. When compared to a pure aluminum shaft, for the non-rotating case, the first frequency increases by $37 \%$, the second by $11 \%$ and the third by $5 \%$. For the rotating case, the first frequency increases by $33 \%$, the second by $10 \%$ and the third by $4 \%$. In this case though, the overall increase in weight is about $95 \%$, which, when compared to the previous FGMs, can impose restrictions on the use of this material.

As a final study, an exploration of the power-law distribution effects on the frequencies and mass of shafts built using this category of materials is carried out in the following. The study was conducted for the ceramic metallic materials only. Figures 2 and 3 show frequency and mass variations, as functions of $\zeta$, for shafts built of alumina/aluminum and zirconia/aluminum respectively. The plots depict trend results for the materials as the distribution between the metallic and ceramic phases is changed. Note that the included tables show the first three frequencies and mass values of the shafts for discrete values of $\zeta$, which varies from 1 to 5 .

For alumina/aluminum, it is seen that all three frequencies increase as $\zeta$ increases. Between $\zeta$ values of 1 and 5 variations are $4.4 \%, 17.3 \%$ and $20.1 \%$ for the first, second and third frequencies, respectively. Note though that 
this is accompanied by a mass increase of approximately $10.3 \%$. This trend is shown in the respective plot which includes extended frequency and mass values.

For zirconia/aluminum results are mixed. Between $\zeta$ values of 1 and 5, the first frequency decreases by $7.1 \%$, the second practically does not vary and the third increases by $1 \%$. In this case the mass increase is about $25.3 \%$. The included plot shows these results for extended values of frequency and mass.

As a summary of results, one should note that increases in frequencies, for the rotating beam problem, can be achieved by the use of FGMs. The approach is followed by variations, which can be significant, in Young's modulus, density and overall weight of the shafts. Thus manufacturing considerations (see references [4,14]), as well as application requirements, will dictate the advantages of the use of one material over the other.

\section{Conclusions}

The use of FGMs for radially rotating cantilever beams can change the natural frequencies of vibration of the system significantly. For the materials considered, it was shown that the use of ceramic - metallic FGMs can lead to advantages in terms of obtaining higher non-rotating and rotating natural frequencies without excessive weight increases.

For example, a shaft made of aluminum silicon carbide led to increases in frequencies varying from $23 \%$ to $43 \%$ when compared to a pure aluminum shaft. That was achieved with only an $8 \%$ increase in shaft overall weight. Another example that used alumina and aluminum led to increases varying from $60 \%$ to $103 \%$, which are encouraging results since they were achieved with an increase in overall weight of only $18 \%$. The choice of materials will then depend on frequency versus weight demands as well as manufacturing issues.

A shaft composed of two metallic phases also led to increases in frequencies but with a larger increase in weight when compared to the ceramic - metallic ones, which shows that the latter category is a better choice.

\section{References}

[1] M. Ahmad and M.N. Naeem, Vibration Characteristics of Rotating Fgm Circular Cylindrical Shells Using Wave Propagation Method, European Journal of Scientific Research 36(2) (2009), 184-235.

[2] R.B. Bhat, Transverse Vibrations of a Rotating Uniform Cantilever Beam with Tip Mass as Predicted by Using Beam Characteristic Orthogonal Polynomials in the Rayleigh-Ritz Method, Journal of Sound and Vibration 105(2) (1986), 199-210.

[3] T.S. Chihara, Introduction to Orthogonal Polynomials, London, Gordon and Breach, 1978.

[4] K.-L. Choy and E. Felix, Functionally Graded Diamond-Like Carbon Coatings on Metallic Substrates, Materials Science and Engineering A(278) (2000), 162-169.

[5] S.A. Fazelzadeh and M. Hosseini, Aerothermoelastic Behavior of Supersonic Rotating Thin-Walled Beams Made of Functionally Graded Materials, Journal of Fluids and Structures 23(8) (2007), 1251-1264.

[6] D.T. Greenwood, Principles of Dynamics, Englewood Cliffs, N. J., Prentice-Hall, Inc., 1988.

[7] W.J. Haering, R.R. Ryan and R.A. Scott, New Formulation for Flexible Beams Undergoing Large Overall Plane Motion, Journal of Guidance, Control and Dynamics 17(1) (1994), 76-83.

[8] S.V. Hoa, Vibration of a Rotating Beam with Tip Mass, Journal of Sound and Vibration 67(3) (1979), 369-381.

[9] D.H. Hodges, An Approximate Formula for the Fundamental Frequency of a Uniform Rotating Beam Clamped Off the Axis of Rotation, Journal of Sound and Vibration 77(1) (1981), 11-18.

[10] D.H. Hodges and M.J. Rutkowski, Free-Vibration Analysis of Rotating Beams by a Variable-Order Finite-Element Method, AIAA Journal 19(11) (1981), 1459-1466.

[11] X.X. Hu, T. Sakiyama, H. Matsuda and C. Morita, Fundamental Vibration of Rotating Cantilever Blades with Pre-Twist, Journal of Sound and Vibration 271 (2004), 47-66.

[12] S.H. Hyun and H.H. Yoo, Dynamic Modelling and Stability Analysis of Axially Oscillating Cantilever Beams, Journal of Sound and Vibration 228(3) (1999), 543-558.

[13] T.R. Kane, R.R. Ryan and A.K. Banerjee, Dynamics of a Cantilever Beam Attached to a Moving Base, Journal of Guidance, Control and Dynamics 10(2) (1987), 139-151.

[14] K.A. Khor and Y.W. Gu, Effects of Residual Stress on the Performance of Plasma Sprayed Functionally Graded Zro/Nicocr Alloy Coatings, Materials Science and Engineering A(277) (2000), 64-76.

[15] H. Kojima, Transient Vibrations of a Beam/Mass System Fixed to a Rotating Body, Journal of Sound and Vibration 107(1) (1986), $149-154$.

[16] C.L. Lee, M.F. Al-Salem and T.G. Woehrle, Natural Frequency Measurements for Rotating Spanwise Uniform Cantilever Beams, Journal of Sound and Vibration 240(5) (2001), 957-961. 
[17] X.-F. Li, A Unified Approach for Analyzing Static and Dynamic Behaviors of Functionally Graded Timoshenko and Euler-Bernoulli Beams, Journal of Sound and Vibration 318(4-5) (2008), 1210-1229.

[18] L. Librescu, S.-Y. Oh, O. Song and H.-S. Kang, Dynamics of Advanced Rotating Blades Made of Functionally Graded Materials and Operating in High-Temperature Field, Journal of Engineering Mathematics 61(1) (2008), 1-16.

[19] S.C. Lin and K.M. Hsiao, Vibration Analysis of a Rotating Timoshenko Beam, Journal of Sound and Vibration 240(2) (2001), 303-322.

[20] L. Meirovitch, Fundamentals of Vibrations, McGraw-Hill, 2001.

[21] Y. Miyamoto, W.A. Kaysser, B.H. Rabin, A. Kawasaki and R.G. Ford, Functionally Graded Materials: Design, Processing and Applications, Springer, 1999.

[22] S.-Y. Oh, L. Librescu and O. Song, Vibration and Instability of Functionally Grade Circular Cylindrical Spinning Thin-Walled Beams, Journal of Sound and Vibration 285(4-5) (2005), 1071-1091.

[23] M.T. Piovan and R. Sampaio, A Study on the Dynamics of Rotating Beams with Functionally Graded Properties, Journal of Sound and Vibration 327 (2009), 134-143.

[24] S. Putter and H. Manor, Natural Frequencies of Radial Rotating Beams, Journal of Sound and Vibration 56(2) (1978), 175-185.

[25] A.G. Rao, M. Mohape, V.A. Katkar, D.S. Gowtam, V.P. Deshmukh and A.K. Shah, Fabrication and Characterization of Aluminum (6061) Boron - Carbide Functionally Gradient Material, Materials and Manufacturing 25(7) (2010), 572-576.

[26] T.H. Tan, H.P. Lee and G.S.B. Leng, Dynamic Stability of Radially Rotating Beam Subjected to Base Excitation, Computer Methods in Applied Mechanics and Engineering 146 (1997), 265-279.

[27] J. Woo and S.A. Meguid, Nonlinear Analysis of Functionally Graded Plates and Shallow Shells, International Journal of Solids and Structures 38(42-43) (2001), 7409-7421.

[28] A. Yigit, R.A. Scott and A.G. Ulsoy, Flexural Motion of a Radially Rotating Beam Attached to a Rigid Body, Journal of Sound and Vibration 121(2) (1988), 201-210.

[29] T.-C. Chiu and F. Erdogan, One-Dimensional Wave Propagation in a Functionally Graded Elastic Medium, Journal of Sound and Vibration 222(3) (1999), 453-487.

[30] www.maplesoft.com. 

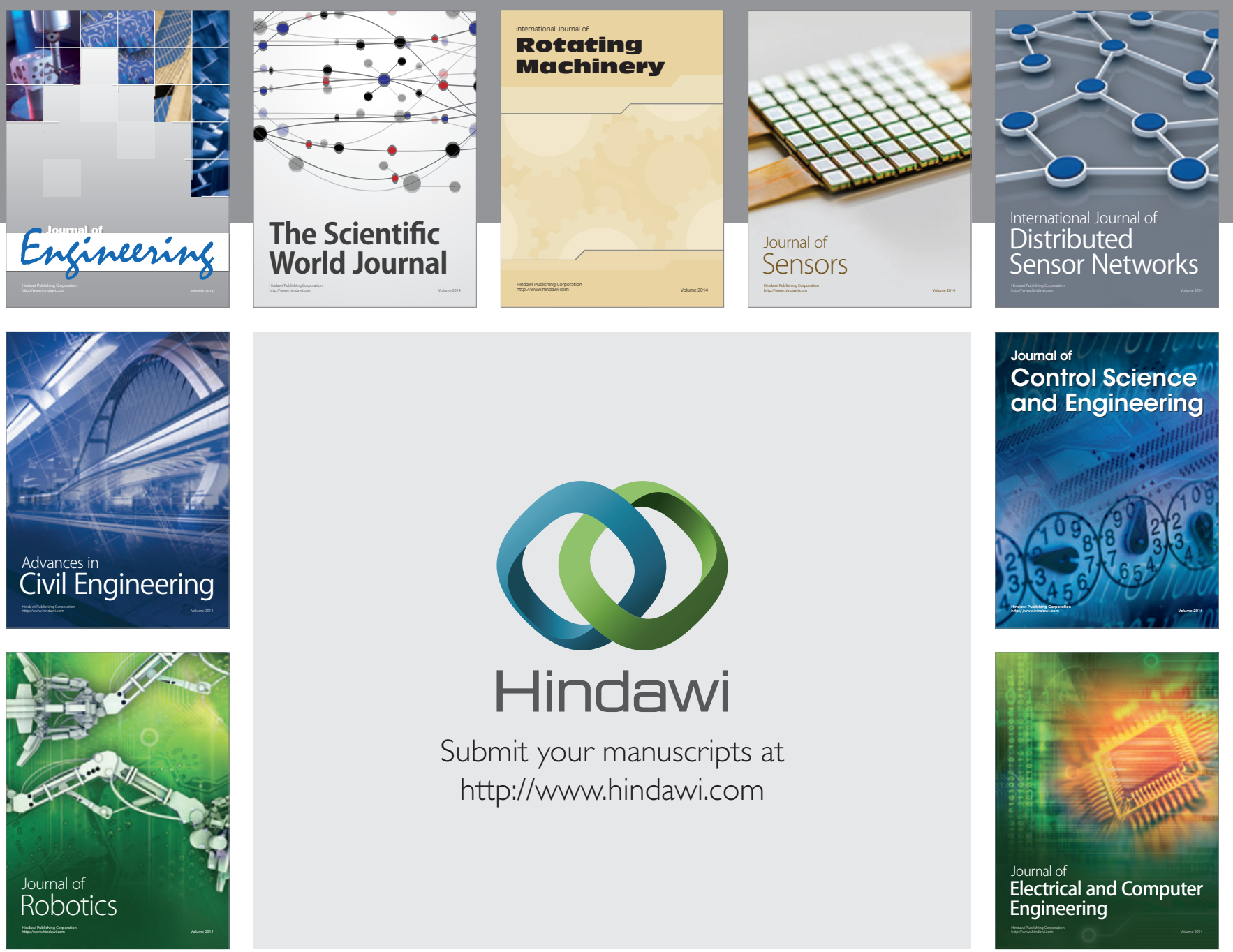

Submit your manuscripts at

http://www.hindawi.com
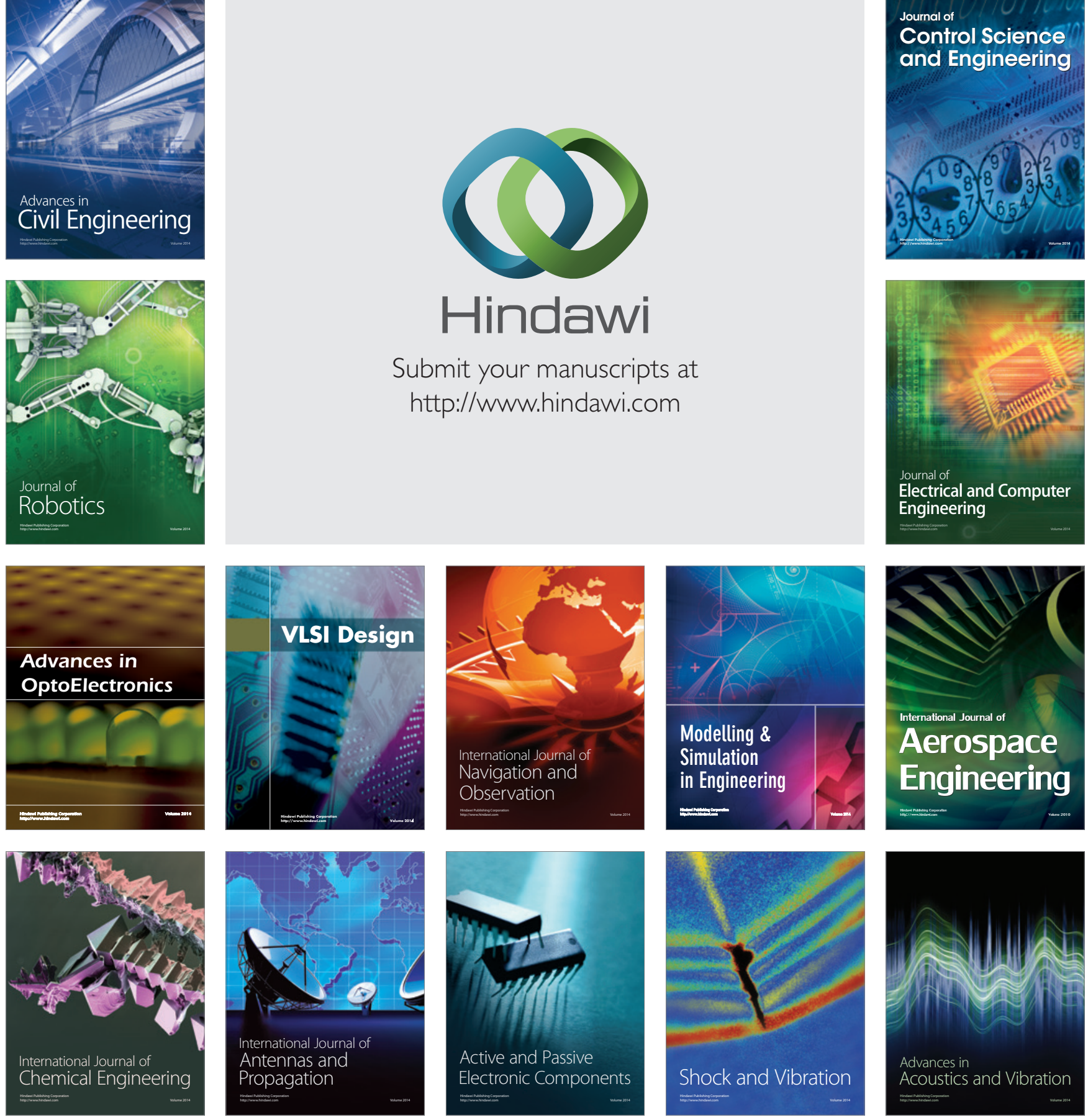